\title{
Prevention of diet-induced obesity by apple polyphenols in Wistar rats through regulation of adipocyte gene expression and DNA methylation patterns.
}

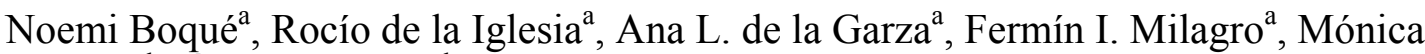
Olivares $^{\mathrm{b}}$, Óscar Bañuelos ${ }^{\mathrm{b}}$, A. Cristina Soria ${ }^{\mathrm{c}}$, Sonia Rodríguez-Sánchez ${ }^{\mathrm{c}}, \mathrm{J}$. Alfredo Martínez ${ }^{\mathrm{a}}$, Javier Campión ${ }^{\mathrm{a}^{*}}$.

a Department of Nutrition, Food Science, Physiology and Toxicology. University of Navarra. 31009, Pamplona. Spain. ${ }^{b}$ Discovery Laboratory. Health and Nutrition Department. Biosearch S.A. 18004, Granada. Spain. ${ }^{\mathrm{c}}$ Department of Instrumental Analysis and Environmental Chemistry. Institute of General Organic Chemistry (CSIC). 28006, Madrid, Spain.

* To whom correspondence should be addressed.

Tel.0034-948-425600 Fax: 0034-948-425649, e-mail address: jcampion@,unav.es

Discovery Laboratory, Health and Nutrition Department, Biosearch S.A., Granada, Spain

Discovery Laboratory. Health and Nutrition Department. Biosearch S.A. Granada. Spain

\section{CORRESPONDING AUTHOR}

Dr. Javier Campión

Department of Nutrition, Food Science, Physiology and Toxicology

University of Navarra

$\mathrm{C} /$ Irunlarrea $\mathrm{s} / \mathrm{n}$

31008 Pamplona, SPAIN

Phone: 0034948425600

Fax: 0034948425649

E-mail address: jcampion@unav.es

\footnotetext{
Abbreviations: AP, apple polyphenols; DIO, diet-induced obesity; HFS, high-fatsucrose; IPGTT, intraperitoneal glucose tolerance test; RQ, respiratory quotient; WAT, white adipose tissue; HOMA, homeostatic model assessment
} 
Key words: high-fat-sucrose diet; adiposity; lipolysis; nutrigenomics; epigenetics.

\begin{abstract}
This study was conducted to determine the mechanisms implicated in the beneficial effects of apple polyphenols (AP) against diet-induced obesity (DIO) in Wistar rats, described in a previous study from our group. Supplementation of high-fat-sucrose (HFS) diet with AP prevented adiposity increase by inhibition of adipocyte hypertrophy. Rats supplemented with AP exhibited improved glucose tolerance while adipocytes isolated from these rats showed an enhanced lipolytic response to isoproterenol. AP intake led to reduced Lep, Plin and Srebf1 mRNA levels and increased Aqp7, Aebpl and Ppargcla mRNA levels in epidydimal adipocytes. In addition, we found different methylation patterns of Aqp7, Lep, Ppargcla and Srebf1 promoters in adipocytes from apple-supplemented rats compared to HFS-fed rats. The administration of AP protects against body weight gain and fat deposition and improves glucose tolerance in rats. We propose that AP exerts the anti-obesity effects through the regulation of genes involved in adipogenesis, lipolysis and fatty acid oxidation, in a process that could be mediated in part by epigenetic mechanisms.
\end{abstract}


Apples are a rich source of polyphenols, which consist of a complex mixture of chlorogenic acid, phloridzin, quercetin, catechin, epicatechin, procyanidin and rutin, among others compounds [1]. These phenolic substances have been reported to display antihyperglycemic, antihyperlipidemic and anti-inflammatory properties [2]. Furthermore, previous studies have demonstrated the effect of apple polyphenols (AP) on fat deposition, by decreasing the weight of visceral adipose tissue in rats fed a control [3] or high fat-diet [4]. In the same way, AP were able to decrease triglyceride absorption by inhibiting pancreatic lipase activity in mice and humans [5]. Our group have recently published a study where supplementation of HFS diet with AP during 8 weeks (700mg/Kg body weight) prevented body weight and adiposity gain (in all fat pads) promoted by this obesogenic diet, without changes in food intake [6]. AP was also found to protect from HFS diet-induced hyperglycemia, hyperleptinemia and insulinresistance.

Since the mechanisms implicated in the effects of AP against DIO have not been well elucidated yet, the purpose of this work was to evaluate changes in cellularity, mRNA expression and DNA methylation induced by the ingestion of AP on rat adipocytes, in order to achieve a deeper understanding of the molecular changes in response to AP supplementation.

Our results from histological studies demonstrated that supplementation of HFS with AP markedly reversed the enlargement of adipocyte volume induced by HFS diet intake (Table 1) in the epididymal fat pad, reducing it by almost $28 \%$, without changes in the subcutaneous one. AP supplementation reversed the increase on the population of 
large epididymal adipocytes, especially with diameters higher than $130 \mu \mathrm{m}$ (Supporting information: Fig. S1).

AP supplementation reversed the increase in blood glucose levels observed in HFS-fed rats after the intraperitoneal glucose tolerance test (IPGTT) (Fig. S3A) with a significant decrease of $14 \%$ in the total area under the curve (Fig. S3B).

We further explored the lipolytic capacity of epididymal adipocytes from animals supplemented with AP (Fig. S4). HFS intake impaired the catecholamineinduced lipolytic activity of adipocytes, with an inhibition of 54\%. Interestingly, supplementation with AP tended to restore the lipolytic response of fat cells to isoproterenol stimulation (HFS $0.42 \pm 0.32$; HFS + AP $0.77 \pm 0.32 \mu$ moles glycerol $/ 100 \mathrm{mg}$ lipids/90 min; $\mathrm{p}=0.065)$.

To examine AP-induced gene expression modifications in isolated epididymal adipocytes, a quantitative RT-PCR primer array was performed (Supporting information: Table S1). Gene expression levels of leptin, perilipin (Plin) and sterol regulatory element binding transcription factor 1 (Srebfl) were upregulated by HFS whereas this effect was normalized by AP supplementation (Fig. 1). Interestingly, some genes were only regulated by AP supplementation (Fig. 1). Thus, the adipocyte enhancer binding protein 1 (Aebpl), aquaporin 7 (Aqp7) and peroxisome proliferatoractivated receptor gamma coactivator 1 alpha (Ppargc 1a) were significantly overexpressed in the supplemented group compared with the HFS group.

After that, we measured the methylation pattern in the promoters of those genes which expression levels had been modified by AP supplementation (Fig. 2 and Supporting information: Fig. S6). HFS intake resulted in hypomethylation of $3 \mathrm{CpG}$ sites of Aqp7 promoter (Fig. 2) whereas supplementation with AP reversed these 
effects. The opposite pattern was found in $2 \mathrm{CpG}$ sites of leptin promoter, with increased methylation in HFS group and significant decreased methylation in AP group. Notably, AP could also modify the percentage of DNA methylation in one CpG site of Srebfl promoter, with a reduction of 52\%, and Ppargcla promoter, with an increase of 14\%. Moreover, we found a significant correlation between Lep_CpG22 methylation degree and leptin gene expression and also between Aqp7 promoter total methylation and Aqp7 expression levels (Fig. 2). Additionally, there was a negative correlation between total Aqp7 methylation and Lep_CpG22 methylation levels. Epidydimal fat mass was negatively correlated with Aqp7_CpG4 methylation while Aqp7_CpG4 percentage methylation was inversely correlated with insulin plasmatic levels and leptin mRNA levels.

Our results obtained in the epidydimal depot indicate that AP prevents adipose tissue accumulation mainly through a prevention of hypertrophy rather than hyperplasia. These effects could be mediated through the increased capacity of these adipocytes to respond to catecholamine-induced lipolysis. In this way, other polyphenols have been reported to decrease $\beta$-adrenergic mediated lipolysis [7]. However, we cannot rule out other mechanisms that could be implicated, as for example inhibition of dietary fat absorption, since it has been observed that polyphenols induce this effect in vivo, probably due to its inhibitory effect on pancreatic lipase activity [5].

In addition, AP supplementation normalizes glucose levels after the IPGTT. In this context, quercetin and phloridzine, polyphenols found in apples, have been reported to decrease intestinal glucose uptake $[8,9]$. The diminished population of larger-sized adipocytes in rats supplemented with AP could explain this improvement of glucose tolerance, as it has been seen that adipocyte size is related with insulin resistance [10] and that large adipocytes exhibit lower insulin-stimulated glucose oxidation [11]. 
Evidence from various studies indicates that polyphenols consumption prevents fat accumulation through the activation of $\beta$-oxidation $[12,13]$. Our results show an upregulation by AP of the key transcription factor Ppargcla, which could be related with an increase in fatty acid oxidation. Concomitantly, the increase in Ppargcla mRNA expression could indicate a partial shift from a white to brown adipose tissue phenotype [14].

On the other hand, Aqp7 is known to modulate adipocyte glycerol permeability thereby controlling triglyceride accumulation and glucose homeostasis. Accordingly, overexpression of Aqp7 could help to explain the increased glycerol released observed in the ex vivo lipolysis experiments and the smaller size of the adipocytes of AP-fed rats, although no changes were found in the mRNA levels of the key lipolytic genes Lipe and Pnpla2.

Another mechanism that could be implicated in the anti-obesity effects of AP is the inhibiton of adipogenesis, as indicated by the enhanced expression of Aebpl [15]. Remarkably, it has been previously described that in preadipocytes, Aebpl mRNA levels are modulated by some plant extracts [16]. Although we did not find changes in key transcription factors related with adipogenesis, like $P P A R \gamma$ or $C E B P \alpha$, the expression levels of Srebfl, which also participates in the control of adipogenesis, were downregulated by AP. In this sense, it has been shown that other polyphenolic extracts inhibited adipocyte differentiation through downregulation of Srebf1 [17]. Furthermore, decreased mRNA levels of perilipin in adipocytes from AP-supplemented rats could indicate again an inhibition of adipogenesis by AP since higher expression of perilipin levels are related with increased adipogenesis and higher lipid droplets size [18]. 
AP supplementation reversed the increased leptin expression levels induced by HFS feeding. The role of leptin in the regulation of energy expenditure and food intake has been clearly evidenced [19]. Results of leptin gene expression are in line with the decreased circulating leptin levels and reflect the reduced fat mass observed in APsupplemented animals.

DNA methylation is involved in the control of gene expression together with other epigenetic processes such as histone modifications and miRNAs [20]. Some studies have evidenced a role of AP on the modulation of DNA methylation in relation with its chemopreventive properties [21]. Our group has reported previously that leptin promoter is modulated by high-fat diet in adipocytes [22]. Now, we describe an increase in leptin CpG (position -292 and -198) metyhlation by HFS diet and a reduction of methylation to normal values by $\mathrm{AP}$ in the $\mathrm{CpG}$ at the position -198. We have also observed an increase of Ppargcla promoter methylation by AP. Other studies have evidenced an epigenetic control of Ppargcla gene expression [23] in human muscle. On the other hand, we have reported for the first time an epigenetic regulation of Aqp 7 gene promoter both by HFS diet and AP and of Srebfl by AP, pointing out these genes as potential candidates for future epigenetic studies.

Aqp7 and Leptin promoter methylation levels were significantly correlated with the corresponding gene expression levels. Methylation of $\mathrm{CpG}$ rich sequences in gene promoter regions usually represses gene expression by interfering with binding of proteins required for transcription. Unexpectedly, we found that DNA methylation was positively correlated with gene expression. However, it has been proposed that if DNA methylation occurs on negative regulatory elements in the promoter, the transcriptional activity could be increased [24]. The inverse correlation observed between Aqp7_CpG4 percentage methylation and insulin levels as well as leptin expression are especially 
relevant because it has been described recently that leptin and insulin directly regulate Aqp 7 expression through the PI3K/Akt/mTOR pathway in cultured adipocytes [25].

Although we have found many beneficial effects induced by AP intake, the dose used is quite high and it is equivalent to a human consumption of $146 \mathrm{mg}$ of $\mathrm{AP}$ per $\mathrm{kg}$ of body weight, accordingly to the formula proposed by Reagan-Shaw S et al [26]. This fact must be taking into account if human intervention studies are designed.

Taken together, our results demonstrated that AP extract exerts potent antiobesogenic and anti-diabetic effects mainly through direct activity on adipocytes modulating $\beta$-adrenergic stimulated lipolysis as well as adipogenic, lipolytic and oxidative-related genes. Furthermore, some of these genes could be subjected to epigenetic regulation. In conclusion, this study highlights AP extract as a promising functional food ingredient for the management of obesity and its metabolic complications.

Fundings: Research related to this work was funded by Biosearch SA in the framework of the PRONAOS project granted by the Center for Industrial Technological Development (CDTI) and by the Asociación de Amigos de la Universidad de Navarra.

Aknowledgments: The technical assistance of Ana Lorente is gratefully acknowledged.

Conflict of interest statement: Dr Olivares and Dr Bañuelos are employees of Biosearch S.A., which provided the plant extracts and research funding for this study. 


\section{REFERENCES}

1. Gerhauser, C., Cancer chemopreventive potential of apples, apple juice, and apple components. Planta. Med. 2008, 74, 1608-24.

2. Pandey, KB., Rizvi, SI., Plant polyphenols as dietary antioxidants in human health and disease. Oxid. Med. Cell. Longev. 2009, 2, 270-8.

3. Nakazato, K., Song, H., Waga, T., Effects of dietary apple polyphenol on adipose tissues weights in Wistar rats. Exp. Anim. 2006, 55, 383-9.

4. Ohta, Y., Sami M., Kanda T., Saito K. et al., Gene Expression Analysis of the Antiobesity Effect by Apple Polyphenols in Rats Fed a High Fat Diet or a Normal Diet. J. Oleo. Sci. 2006, 55, 305-14.

5. Sugiyama, H., Akazome Y., Shoji T., Yamaguchi A. et al., Oligomeric procyanidins in apple polyphenol are main active components for inhibition of pancreatic lipase and triglyceride absorption. J. Agric. Food. Chem. 2007, 55, 46049.

6. Boqué, N., Campión, J., de la Iglesia, R., de la Garza, A.L. et al. Screening of polyphenolic plant extracts for anti-obesity properties in Wistar rats. J. Sci. Food. Agric. 2012, In press.

7. Ogasawara, J., et al, Comparison of the effect of oligonol, a new lychee fruitderived low molecular form of polyphenol, and epigallocatechin-3-gallate on lipolysis in rat primary adipocytes. Phytother. Res. 2011, 25, 467-71.

8. Manzano, S., Williamson, G., Polyphenols and phenolic acids from strawberry and apple decrease glucose uptake and transport by human intestinal Caco-2 cells. Mol. Nutr. Food. Res. 2010, 54, 1773-80. 
9. Zhao, H., et al, Phloridzin improves hyperglycemia but not hepatic insulin resistance in a transgenic mouse model of type 2 diabetes. Diabetes 2004, 53, 29019.

10. Jernas, M., et al, Separation of human adipocytes by size: hypertrophic fat cells display distinct gene expression. Faseb. J. 2006, 20, 1540-2.

11. Salans, L.B., Dougherty, J.W., The effect of insulin upon glucose metabolism by adipose cells of different size. Influence of cell lipid and protein content, age, and nutritional state. J. Clin. Invest. 1971, 50, 1399-410.

12. Caimari, A., Del Bas, J.M., Crescenti, A., Arola, L. Low doses of grape seed procyanidins reduce adiposity and improve the plasma lipid profile in hamsters. Int. J. Obes. (Lond). 2012, In press.

13. Wang, L., Yamasaki, M., Katsube, T., Sun, X. et al. Shiwaku K.Antiobesity effect of polyphenolic compounds from molokheiya (Corchorus olitorius L.) leaves in LDL receptor-deficient mice. Eur. J. Nutr. 2011, 50,127-33.

14. Karamanlidis, G., Karamitri A., Docherty K., Hazlerigg D.G. et al., C/EBPbeta reprograms white 3T3-L1 preadipocytes to a brown adipocyte pattern of gene expression. J. Biol. Chem. 2007, 282, 24660-9.

15. Kim, S.W., Muise, A.M., Lyons, P.J., Ro, H.S., Regulation of adipogenesis by a transcriptional repressor that modulates MAPK activation. J. Biol. Chem. 2001, 276, 10199-206.

16. Yamakawa, J., Ishigaki Y., Takano F., Takahashi T. et al., The Kampo medicines Orengedokuto, Bofutsushosan and Boiogito have different activities to regulate gene expressions in differentiated rat white adipocytes: comprehensive analysis of genetic profiles. Biol. Pharm. Bull. 2008, 31, 2083-9. 
17. Park, H.J., Della-Fera M.A., Hausman D.B., Rayalam S. et al., Genistein inhibits differentiation of primary human adipocytes. J Nutr. Biochem. 2009, 20, 140-8.

18. Park, J.R., Jung J.W., Seo M.S., Kang S.K. et al., DNER modulates adipogenesis of human adipose tissue-derived mesenchymal stem cells via regulation of cell proliferation. Cell. Prolif. 2010, 43, 19-28.

19. Masaki, T., et al, Corticotropin-releasing hormone-mediated pathway of leptin to regulate feeding, adiposity, and uncoupling protein expression in mice. Endocrinology 2003, 144, 3547-54.

20. Campion, J., Milagro, F.I., Martinez, J.A., Individuality and epigenetics in obesity. Obes. Rev. 2009, 10, 383-92.

21. Fini, L., Piazzi G., Daoud Y., Selgrad M. et al., Chemoprevention of intestinal polyps in ApcMin/+ mice fed with western or balanced diets by drinking annurca apple polyphenol extract. Cancer. Prev. Res. (Phila) 2011, 4, 907-15.

22. Milagro, F.I., Campion J., Garcia-Diaz D.F., Goyenechea E. et al., High fat dietinduced obesity modifies the methylation pattern of leptin promoter in rats. $J$. Physiol. Biochem. 2009, 65, 1-9.

23. Brons, C., Jacobsen S., Nilsson E., Ronn T. et al., Deoxyribonucleic acid methylation and gene expression of PPARGC1A in human muscle is influenced by high-fat overfeeding in a birth-weight-dependent manner. J. Clin. Endocrinol. Metab. 2010, 95, 3048-56.

24. Ngo, V., Gourdji, D., Laverriere, J.N., Site-specific methylation of the rat prolactin and growth hormone promoters correlates with gene expression. Mol. Cell. Biol. $1996,16,3245-54$.

25. Rodriguez, A., Catalan V., Gomez-Ambrosi J., Garcia-Navarro S. et al., Insulinand leptin-mediated control of aquaglyceroporins in human adipocytes and 
hepatocytes is mediated via the $\mathrm{PI} 3 \mathrm{~K} / \mathrm{Akt} / \mathrm{mTOR}$ signaling cascade. J. Clin.

Endocrinol. Metab. 2011, 96, E586-97.

26. Reagan-Shaw, S., Nihal, M., Ahmad, N., Dose translation from animal to human studies revisited. FASEB. J. 2008, 22, 659-61. 


\section{FIGURES CAPTIONS}

Figure 1. Effect of apple polyphenols (AP) on mRNA levels in isolated epidydimal adipocytes from male Wistar rats fed with high-fat-sucrose (HFS) diet supplemented with or without AP for 56 days. Results were normalized to GAPDH mRNA levels and expressed as fold change respect to controls (control set at unit). Data are means $\pm \mathrm{SE}$ $(\mathrm{n}=5-6) ; * * \mathrm{p}<0.01$ vs control group. $\# \mathrm{p}<0.05, \# \# \mathrm{p}<0.01$ vs HFS group. See supporting information: Table S1.

Figure 3. Effect of apple polyphenols (AP) on the percentage of methylation (A) of specific $\mathrm{CpG}$ dinucleotides in gene promoters of isolated epidydimal adipocytes from male Wistar rats fed with high-fat-sucrose (HFS) diet supplemented with or without AP for 56 days. (B) Correlation analyses between percentage of DNA methylation and gene expression levels (R, Pearson's correlation coefficient). Data are means $\pm \mathrm{SE}(\mathrm{n}=5-6)$; * $\mathrm{p}<0.05,{ }^{* *} \mathrm{p}<0.01$ vs control group. $\# \mathrm{p}<0.05, \# \# \mathrm{p}<0.01$ vs HFS group. See supporting information: Fig. S6. 